The concentration of soluble IL- 6 receptor increased after BCD-089 administration in a dose-dependent manner. A raise in the concentration of IL- 6 was seen at doses $>1.0 \mathrm{mg} / \mathrm{kg}$. Membrane IL-6R saturation of $90 \%>100 \%$ was seen at doses $\geq 0.6 \mathrm{mg} / \mathrm{kg}$. Regardless to the dose, serum CRP decreased below the limit of detection in most volunteers within the first week after injection. All tested PD markers returned to baseline at the end of the follow-up.

Conclusions: Single SC administration of BCD-089 was well tolerated, showed favourable safety profile and low immunogenicity at all tested doses in healthy volunteers. PK/PD assessment revealed non-linear PK and significant capacity to inhibit the IL-6 signalling pathway. These finding supports further clinical development of BCD-089.

Disclosure of Interest: P. Khlyabova: None declared, A. Eremeeva Employee of: JSC BIOCAD, A. Lutckii Employee of: JSC BIOCAD, E. Dokukina Employee of: JSC BIOCAD, E. Chernyaeva Employee of: JSC BIOCAD, R. Ivanov Employee of: JSC BIOCAD

DOI: 10.1136/annrheumdis-2018-eular.2410

\section{SAT0024 IL-36 AXIS IS AN EMERGING THERAPEUTIC TARGET IN PSORIATIC ARTHRITIS SYNOVIAL TISSUE}

M.-A. Boutet, A. Nerviani, G. Lliso-Ribera, K. Goldmann, M. Lewis, C. Pitzalis. Centre for Experimental Medicine and Rheumatology, Queen Mary University, London, UK

Background: The IL-36 family of cytokines includes three agonists, IL-36 $\alpha$, IL$36 \beta$ and IL-36 $\gamma$, and two established or hypothetical antagonists, respectively IL36Ra and IL-38. IL-36 agonists are pro-inflammatory cytokines highly expressed in skin and involved in the pathogenesis of psoriasis. A recent study demonstrated that only a subset of patients with rheumatoid arthritis (RA) had an elevated IL-36 agonists/antagonists ratio within the synovium and could potentially respond to IL36 inhibition strategies but little is known about the expression and biologic functions of the IL-36 axis in synovial tissue of psoriatic arthritis (PSA) so far hardly been studied.

Objectives: In this study, we aimed to comparatively investigate the expression and role of IL-36 cytokines in synovial tissue of early RA and PsA patients.

Methods: Synovial tissue samples were collected from patients with early RA and PsA (disease duration <12 months) DMARDs (Disease Modifying Anti-Rheumatic Drugs) and steroids-naïve. All patients underwent an ultrasound-guided synovial biopsy before starting the treatment; the procedure was repeated following six months of treatment with conventional DMARDs. The expression of IL-36 family members was investigated in synovial tissue at gene level by RNASequencing (87 RA, $15 \mathrm{PsA}$ ), at protein level by immunohistochemistry (IHC) and immunofluorescence (IF) (20 RA, 26 PsA) and in plasma by ELISA (22 RA, 38 PSA). RA and PSA-fibroblasts-like-synoviocytes (FLS) and peripheral blood mononuclear cells (PBMCs) were treated in vitro to assess their response to IL-36 stimulation.

Results: Gene and protein expression of IL-36 agonists was comparable between RA and PsA synovial tissue; conversely, the antagonists IL-36Ra and IL38 were significantly lower in PsA compared to RA. Accordingly, the agonists/ antagonists ratio was considerably higher in PsA synovium, suggesting an activation of the IL36 pro-inflammatory pathway. Among the immune cells infiltrating the PsA synovium, macrophages (CD68+), T lymphocytes (CD3+) and plasma cells (CD138+) were the primary IL36 $\alpha$-expressing cells. At baseline, the synovial expression of IL-36 $\alpha$ was significantly higher in PsA patients who did not respond to DMARDs treatment at 12 months; this differential synovial expression of IL-36 $\alpha$ between responders and non-responders was also maintained at six months. In keeping with this observation, we showed that treatment with methotrexate or sulfasalazine did not reduce the expression of IL-36 in PsA cells in vitro. Finally, we observed that PSA-FLS and PSA-PBMCs produced significantly higher levels of IL-8 upon stimulation with IL-36 $\alpha$ in comparison with cells isolated from RA patients.

Conclusions: The expression of the anti-inflammatory IL-36 cytokines antagonists are differently regulated in early RA and PsA, being significantly lower in the latter. Moreover, the pro-inflammatory IL-36 $\alpha$ is up-regulated in synovial tissue of PsA non-responders to conventional DMARDs. The impaired balance between agonists and antagonists might contribute to the persistent inflammation characterising the diseased tissue. The exogenous replacement of the IL-36 antagonists may be a novel promising therapeutic target for PsA patients.

Disclosure of Interest: None declared

DOI: 10.1136/annrheumdis-2018-eular.3485

\section{SAT0025 \\ CXCL4 ALTERS TRANSCRIPTOMIC AND EPIGENETIC IMPRINTING OF DENDRITIC CELLS THEREBY DRIVING FIBROSIS THROUGH EXTRACELLULAR MATRIX FORMATION}

S.C. Sllva-Cardoso ${ }^{1}$, W. Tao ${ }^{1}$, C. Angiolillili , A. P.P. Lopes ${ }^{1}$, C. Bekker ${ }^{1}$, J. van Laar $^{2}$, E. Hack ${ }^{3}$, M. Boes ${ }^{4}$, A. Pandit ${ }^{1}$, T.R.D.J. Radstake ${ }^{1} .{ }^{1}$ Laboratory of Translational Immunology And Department of Rheumatology and Clinical Immunology, ${ }^{2}$ Department of Rheumatology and Clinical Immunology; ${ }^{3}$ Laboratory of Translational Immunology; ${ }^{4}$ Department of Pediatrics, University Medical Center, Utrecht, Utrecht, Netherlands

Background: Aberrant accumulation of extracellular matrix (ECM) in multiple organs or fibrosis is one of the three hallmarks that characterises the pathogenesis of systemic sclerosis (SSc), together with immune dysregulation and small vessel vasculopathy. Recent studies have shown that CXCL4 (Chemokine CXC motif ligand 4) levels are increased in patients with SSc and correlated with skin and lung fibrosis. ${ }^{1}$ CXCL4 plays key role in physiological processes, although it has been also implicated in several pathological conditions such as autoimmune diseases and cancer. We and others have shown that CXCL4 modulates the phenotype and function of immune cells ${ }^{2}$, suggesting a critical role of this chemokine in innate and adaptive immune responses. However, how CXCL4 exactly modulates immune cell responses remains unclear.

Objectives: Here we investigated the impact of CXCL4 exposure on the transcriptome and DNA methylation of monocyte-derived dendritic cells (moDCs), and the consequence on their function.

Methods: We differentiated human moDCs in the presence of CXCL4. After 6 days differentiation, cells were stimulated with a TLR3 ligand (polyl:C) as described in our previous study. ${ }^{2}$ RNA sequencing and DNA methylation profiling was performed at various time points during differentiation and stimulation.

Results: Integration of high-throughput analyses of RNA sequencing and DNA methylation reveals that CXCL4 drives to dramatic changes on the transcriptome and epigenome levels. This is reflected in the dysregulation of critical innate and adaptive immune pathways, like antigen presentation, and cytokine signalling For the first time, we show that CXCL4 potentiates a novel function to dendritic cells, namely, the production of ECM molecules, such as fibronectin (FN1) and osteopontin (OPN). Furthermore, we also found that CXCL4 exposure results in epigenetic imprinting during moDC differentiation. Using novel bioinformatic methods, we have found that CXCL4 mediates the altered cell function via key transcriptional regulators.

Conclusions: This study provides better understanding how CXCL4 affects moDCs through several immune and non-immune pathways and shows for the first time the direct implication of CXCL4 on the production of ECM by inflammatory cells, thereby underscoring the pivotal role of CXCL4 in inflammatory and fibrotic conditions such as SSc.

\section{REFERENCES}

[1] L. van Bon, Affandi AJ, et al. Proteome-wide analysis and CXCL4 as a biomarker in systemic sclerosis. N. Engl. J. Med 2014. doi:10.1056/ NEJMoa1114576

[2] Silva-Cardoso SC, Affandi AJ, et al. CXCL4 Exposure Potentiates TLR Driven Polarization of Human Monocyte-Derived Dendritic Cells and Increases Stimulation of T Cells. J. Immunol 2017. doi:10.4049/ jimmunol. 1602020

Acknowledgements: This study was supported by the: PhD fellowship SFRH/ BD/89643/2012 from the Portuguese FundaÇão para a Ciência e a Tecnologia (FCT) to Sandra C. Silva-Cardoso; China Scholarship Council (CSC) fellowship No. 201606300050 to Weiyang Tao; ERC starting grant (CIRCUMVENT) and Arthritis foundation grant to Timothy R.D.J. Radstake.

Disclosure of Interest: None declared

DOI: 10.1136/annrheumdis-2018-eular.3327

\section{SAT0041 COMPARISON OF DIFFERENT TYPE 1 IFN SIGNATURES DEMONSTRATES CONCORDANCE IN A REAL WORLD, HOME MONITORED SYSTEMIC LUPUS ERYTHEMATOSUS COHORT}

M. Abedi, F. Flores, P. Naranatt, J. Spangler, L. Pan, R. Terbrueggen. Dxterity, Rancho Dominguez, USA

Background: Type 1 Interferon (IFN) expression has been shown to correlate with disease severity in systemic lupus erythematosus (SLE) patients ${ }^{1}$ and antiIFN biologics are being evaluated in a clinical setting. ${ }^{2}$ The 4-gene IFN response signature of IFI27, IFI44, IFI44L and RSAD2 ${ }^{2}$ is frequently used to determine IFN expression. However, numerous studies have reported on the use of different gene signatures. ${ }^{3,4,5}$ The impact of using different IFN gene signatures to stratify SLE patients is unclear. 